\title{
The other side of comparative genomics: genes with no orthologs between the cow and other mammalian species Raffaele Mazza*†1, Francesco Strozzi ${ }^{\dagger 2}$, Andrea Caprera ${ }^{3}$, Paolo Ajmone-Marsan ${ }^{1}$ and John L Williams ${ }^{2}$
} \author{
Codazza, 26900 Lodi, Italy \\ Email: Raffaele Mazza* - raffaele.mazza@unicatt.it; Francesco Strozzi - francesco.strozzi@tecnoparco.org; \\ Andrea Caprera - andrea.caprera@tecnoparco.org; Paolo Ajmone-Marsan - paolo.ajmone@unicatt.it; \\ John L Williams - john.williams@tecnoparco.org \\ * Corresponding author †Equal contributors
}

Address: ${ }^{1}$ Istituto di Zootecnica, Università Cattolica del Sacro Cuore, via Emilia Parmense 84, 29100 Piacenza, Italy, ${ }^{2}$ IDRA Lab, Parco Tecnologico Padano, via Einstein, Loc Cascina Codazza, 26900 Lodi, Italy and ${ }^{3}$ CERSA, Parco Tecnologico Padano, via Einstein, Loc Cascina

Published: I4 December 2009

BMC Genomics 2009, 10:604 doi:10.1/86/147|-2164-10-604
Received: 13 July 2009

Accepted: 14 December 2009

This article is available from: http://www.biomedcentral.com/I47/-2/64/I0/604

(C) 2009 Mazza et al; licensee BioMed Central Ltd.

This is an Open Access article distributed under the terms of the Creative Commons Attribution License (http://creativecommons.org/licenses/by/2.0), which permits unrestricted use, distribution, and reproduction in any medium, provided the original work is properly cited.

\begin{abstract}
Background: With the rapid growth in the availability of genome sequence data, the automated identification of orthologous genes between species (orthologs) is of fundamental importance to facilitate functional annotation and studies on comparative and evolutionary genomics. Genes with no apparent orthologs between the bovine and human genome may be responsible for major differences between the species, however, such genes are often neglected in functional genomics studies.
\end{abstract}

Results: A BLAST-based method was exploited to explore the current annotation and orthology predictions in Ensembl. Genes with no orthologs between the two genomes were classified into groups based on alignments, ontology, manual curation and publicly available information. Starting from a high quality and specific set of orthology predictions, as provided by Ensembl, hidden relationship between genes and genomes of different mammalian species were unveiled using a highly sensitive approach, based on sequence similarity and genomic comparison.

Conclusions: The analysis identified $3,80 \mathrm{I}$ bovine genes with no orthologs in human and 1010 human genes with no orthologs in cow, among which $4 \mathrm{II}$ and 43 genes, respectively, had no match at all in the other species. Most of the apparently non-orthologous genes may potentially have orthologs which were missed in the annotation process, despite having a high percentage of identity, because of differences in gene length and structure. The comparative analysis reported here identified gene variants, new genes and species-specific features and gave an overview of the other side of orthology which may help to improve the annotation of the bovine genome and the knowledge of structural differences between species.

\section{Background}

With the rapid increase in the amount of genome sequence data available, the automated identification of orthologous genes between species becomes of fundamental importance to facilitate functional annotation and for comparative or evolutionary genomics. Homologous 
proteins descend from a common ancestor and may be classified as either orthologous (orthologs) or paralogous (paralogs)[1]. Orthologs are commonly defined as the functional equivalent genes between species, which may have diverged after a speciation event, whereas genes created by a duplication event, either before species divergence (out-paralogs) or after a speciation event (inparalogs), are known as paralogs. Orthologs typically retain similar domain architecture and function and such conservation is an important component in comparative analysis as well as in the annotation of proteins of unknown function, in the characterization of gene function, for evolutionary genomics and the identification of conserved regulatory elements. In contrast, paralogs may have diverged significantly and acquired new functions, e.g. through point mutations or recombination between domains [1], even though a recent review by Studer and Robinson-Rechavi [2] proposed a model in which both orthologs and paralogs diverge in proportion to time of the duplication event and therefore functional changes can occur for both.

Complete and precise delineation of protein coding genes in the genome and the process of assigning gene orthology remains a challenging task in mammalian genomes because of their large size, the difficulty of creating accurate gene models, the complexity of protein domain architecture and the high frequency of gene duplication events, that create large gene families. Errors in ortholog predictions can significantly affect downstream analyses; as a result there has been increasing interest in high quality ortholog prediction techniques.

During the last decade, there have been several methods proposed for routine generation of genome-wide orthology descriptions, which rely mainly on phylogeny, homology or integrated approaches. Orthology detection methods, based mainly on phylogeny, and implemented in software such as RIO [3], Orthostrapper [4,5], PhiGs [6], PhyOP [7], TreeFam [8], or based on evolutionary distance (RSD $[9,10])$, generally do not erroneously report genes as orthologous (false positive, FP), but have a high frequency of missed orthologs (false negative, FN). However, it is difficult to automate phylogenetic analysis approaches for genome-wide analysis, therefore prediction of orthologs for large genome datasets has been typically performed using homology based methods which compare reciprocal-best-BLAST-hits (RBH).

The most frequently used BLAST-based homology methods for detecting orthologous genes include those of BLASTP [11], COG (Cluster of Orthologous Groups [12]), KOG (euKaryotic Orthologous Groups [13]), The Institute for Genomic Research (TIGR) EGO/TOGA database [14], InParanoid/MultiParanoid [15-17], TribeMCL [18],
OrthoMCL [19,20], KEGG Orthology [21], Roundup [10], MSOAR [22], the OMA project [23] and HomoloGene [24]. However, homology methods used to infer orthology often have high FP error rates and low FN error rates, such as observed with BLASTP, where orthology "hits" typically include true orthologs but also many false positive results [25], the later including paralogs and members of gene families. Moreover, the BLAST searches often return, as the highest scoring hit, a protein that is not the nearest phylogenetic neighbour of the query sequence [26]. In summary, phylogeny-based methods are characterized by high specificity and BLAST-based methods by high sensitivity.

To bypass the limits of single phylogeny or homology methods, in this work we used the Ensembl orthology prediction pipeline as reference [27]. Ensembl uses an integrated approach starting from a homology-based method which builds gene-models using species-specific known sequences and proteins from other species aligned to the target genome. All annotated transcripts are based on experimental evidence and the automated pipelines rely on the mRNAs, ESTs and protein sequences submitted into public databases by the scientific community. Therefore, Ensembl does not annotate genes for which there is no prior evidence of a transcribed sequence. Next, the gene orthology and paralogy predictions are generated using a bioinformatic pipeline where maximum likelihood phylogenetic gene trees (generated by TreeBeST) play a key role. This method produces trees that are the most consistent with the conservation of synteny between species and gives fewer anomalous topologies than single protein-based phylogenetic methods [28]. The Ensembl method does not provide a complete gene set for each species, as it has been demonstrated by genome annotation with different methods that have been able to identify many genes in addition to those annotated by the Ensembl automated pipeline, e.g. 700 chicken genes in addition to the Ensembl gene list [29] and more than 1,000 additional genes between mouse and human genome [30].

The current cattle genome sequence (Btau version 4.0) was based on about $7 \times$ genome coverage with $90 \%$ of the total sequence placed on the 29 autosomes and X chromosome. This last release was assembled by creating sequence contigs arranged into scaffolds, on the basis of sequence overlap and BAC ends contig data. The scaffolds were then placed on chromosomes and ordered using BAC and radiation hybrid physical maps. Most sequence contigs remain unchanged from the previous release (Btau3.1), but scaffold assembly was improved. Automated annotation identified about 22,000 genes, with a core set of 14,345 orthologs found between cattle and seven mammalian species [31]. Over 4,000 genes were 
also manually annotated and orthology prediction with dog, human, mouse, rat, opossum, and platypus genomes was resolved for more than $75 \%$ of the predicted bovine genes.

In this work we developed a BLAST-based method to explore the current annotation of the bovine genome and to describe those genes that were classified as being nonorthologous between bovine and other mammalian species (human, mouse and dog), according to Ensembl classification. We used a double comparative approach to identify a set of bovine genes that had no orthologs in the other three mammalian species and a set of orthologs in human, mouse and dog with no bovine counterparts. Within the first set we expected to find genes with speciesspecific features, assembly and annotation problems in addition to bovine specific genes, the so called orphan genes [32]. These are coding sequences having no matches with genes of other annotated species. The orphan genes arose mainly from duplication events, and recent experimental evidence describes them as fast evolving genes $[33,34]$. The second set contains genes that appear to be absent from the bovine genome, while being present in the other species. Alignments, ontology, manual curation and publicly available information were exploited to classify and investigate groups of genes. Differences between species and possible species-specific features or problems in the assembly of genomes were investigated in order to explain missing orthologs.

\section{Results}

\section{In silico libraries set up}

The full gene sets for cow, human, mouse and dog, were downloaded from Ensembl release 50 to a local database, and comprised 22,836, 36,396, 28,329 and 23,550 genes, respectively. The data used here consisted of genes found to be non-orthologous between cow and human which were further filtered to constitute a core of mammalian orthologs by adding to the comparison the gene sets of mouse and dog, currently the most complete mammalian genomes in terms of sequence information $[35,36]$. The initial query in Ensembl 50 using the Biomart tool returned 5,507 bovine genes with no orthologs in human and 19,811 human genes with no orthologs in cow. The high number of human non-orthologous genes is explained by the larger number of annotated genes in the human genome. Mouse and dog gene sets, as described in the method section, were then added to the query which retrieved 3,801 bovine genes with no orthologs in human, mouse and dog, and 1,010 human genes with no orthologs in cow but with orthologs in mouse and dog, as represented in the Venn diagram in Figure 1. The two queries are slightly different in principle because the final cow dataset will contain bovine orphan genes, while the human dataset includes genes that can be considered core mammalian orthologs, apparently missing in cow. These two resulting in silico data libraries were the subjects for a two-way comparison based on the alignment of the genes from a library of one species to the whole genome sequence of the other species; i.e. bovine genes were aligned to the human genome ("cow vs. human comparison") and human genes to the bovine genome ("human vs. cow comparison").

\section{Sequence alignments to genomes}

The longest transcript (canonical transcript) for each nonorthologous gene from the starting libraries was aligned to the genome of the other species, and then the canonical transcripts grouped in six distinct categories; four containing the genes that could be aligned to the genome and two containing the unaligned genes or those aligning with unassembled contigs. The categories with aligned genes are illustrated schematically in Figure 2. The "potential ortholog" category (Figure 2a) included all the sequences that fully or partially overlapped exons of known genes on the genome, and which might represent orthologs missed by the Ensembl pipeline. The genes that could be aligned to intergenic regions of the genome were included in the "new gene" category (Figure 2b) and might be considered a source for putative new species-specific genes identified in the genome. The "gene variant" category (Figure 2c) included all the sequences that overlapped known exons but also aligned to unannotated or intronic regions that may indicate immature transcripts, transcript variants or different gene structures. Finally, genes that were completely aligned within one or more introns of a known gene were classified as "intronic" (Figure 2d) and probably constitute nested genes. All the genes not producing a significant alignment with the genome or aligned to unassembled contigs, where the annotation was not available, were classified respectively as "not aligned" and "contig".

The 3,801 bovine genes obtained from the "cow vs. human" comparison, were aligned to the human genome: $3,390(89 \%)$ were mapped with a variable degrees of identity, while $411(11 \%)$ sequences had no hit on the human genome. The statistical box plots in Figure 3a show that the median percentage identity of the best hits was about $80 \%$, while the lowest value was around $53 \%$, with an E-value lower than 5e-46, indicating highly significant alignments. Table 1 (column "cow vs. human") shows that 2,533 sequences $(67 \%)$ were identified that overlapped known genes on the human genome, 472 $(12 \%)$ fell into the class "new gene" and $289(8 \%)$ were classified as "gene variant".

The same procedure was carried out for the "human vs. cow" comparison; 967 (96\%) human sequences out of 1,010 were mapped to the bovine genome. While 43 (4\%) 

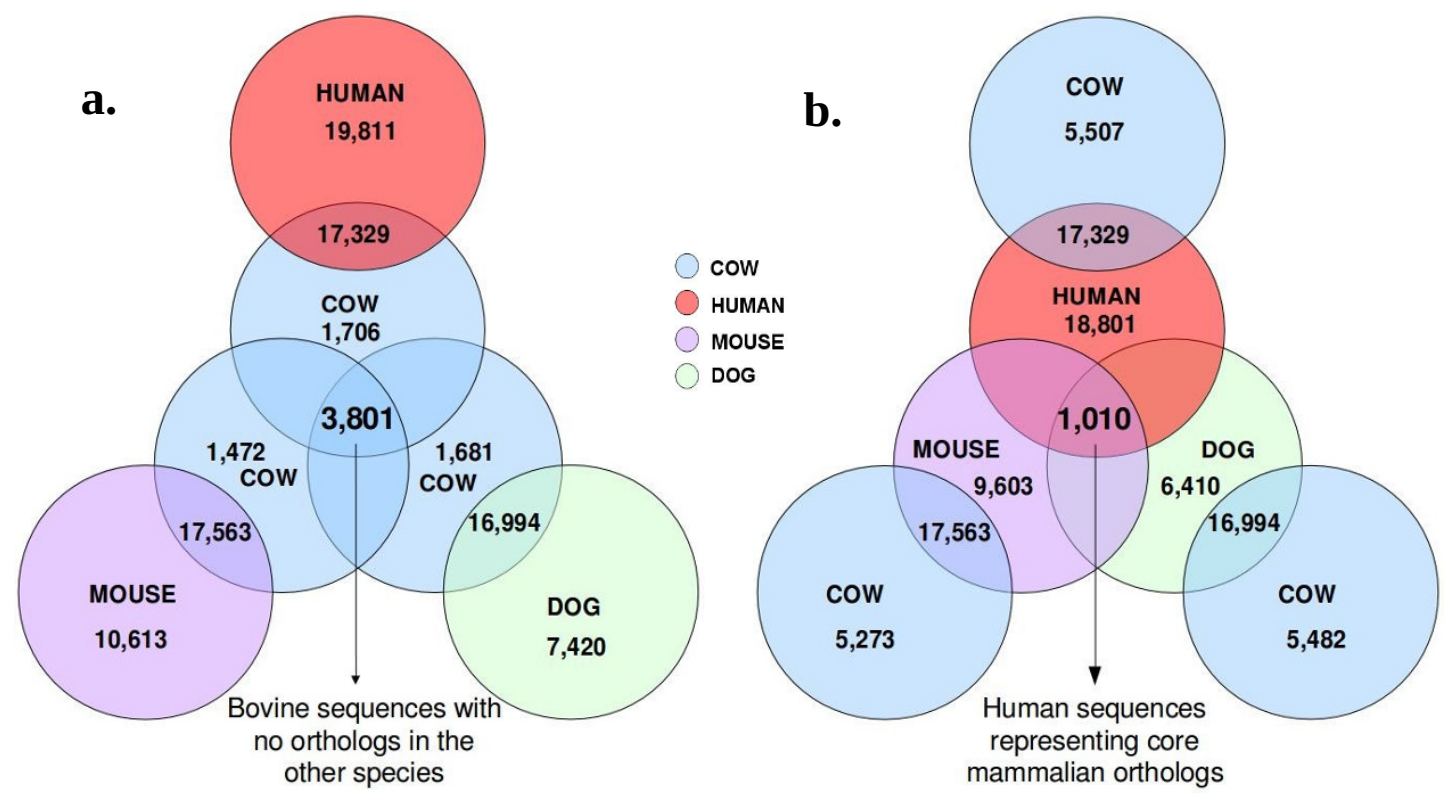

\section{Figure I}

Venn diagram representation of the results obtained from the queries in Ensembl release 50. Each colored circle represents a gene set for a specie. a) query result returning 3,80I cow genes with no orthologs in human, mouse and dog. b) query results returning $\mathrm{I}, 0 \mathrm{I} 0$ human genes representing core mammalian orthologs having no orthologs in cow but with orthologs in mouse and dog.

had no hit at all, the statistical box plots in Figure 3b show that the median percentage of identity for the best hit of the mapped sequences on the genome is about $77 \%$ and does not fall below 55\%, while the E-value is always below 2.6e-54. The aligned sequences were divided into the six categories described above (Table 1, column "human vs. cow"); 120 sequences could be aligned to known bovine genes ("potential ortholog" category). A considerable number of sequences fell into "new gene" and "gene variant" groups (500 and 294, respectively).

\section{Analysis of alignments}

All the bovine and human genes were classified by Gene Ontology (GO) terms and the GO tree was displayed with the web tool described in the gene ontology analysis section of the methods (Figure 4, [37]). The tree for the three main roots (molecular function, biological process and cellular component) was built for the whole set of sequences and for each of the five categories in which the sequences were classified according to the alignment results. Using this web tool the user can navigate through the tree and for each level can retrieve all the sequences described by a specific GO term. In addition, the tool has a direct link to the Ensembl gene viewer and, for bovine sequences, a link to the BLAST best protein hit at NCBI.

In cow, few GO terms were available for the bovine gene set and, even though a similarity search approach was used to provide new terms, about $36 \%(1,382)$ of the sequences, mainly from the "potential ortholog" (918) and "new gene" (310) libraries, could not be included in the tree. The majority of the sequences in the molecular function root were described as proteins with binding properties for other proteins, ions and nucleic acids. Other consistent classes contained genes involved in "catalytic activities" such as hydrolases, transferases and oxidoreductases or signal transducers.

A GO term could not be retrieved for about 10\% (96) of human genes, mainly (67) coming from the "new gene" library. The distribution of the sequences in the human trees resembled those observed in cow with minor differences.

The biotype classification in Ensembl described all the 1,010 human genes as protein coding genes. However, among the 3,801 genes constituting the bovine libraries, a large number $(1,114)$ was represented by different species of RNA (miRNA, rRNA, snRNA and snoRNA), while those remaining were annotated as protein coding $(2,001)$, pseudogene (559) and retrotransposed genes (127). About $86 \%(1,728)$ of the protein coding genes could be aligned to the human genome.

As expected by the different level of annotation between the two species, $66 \%$ of the bovine protein coding genes 

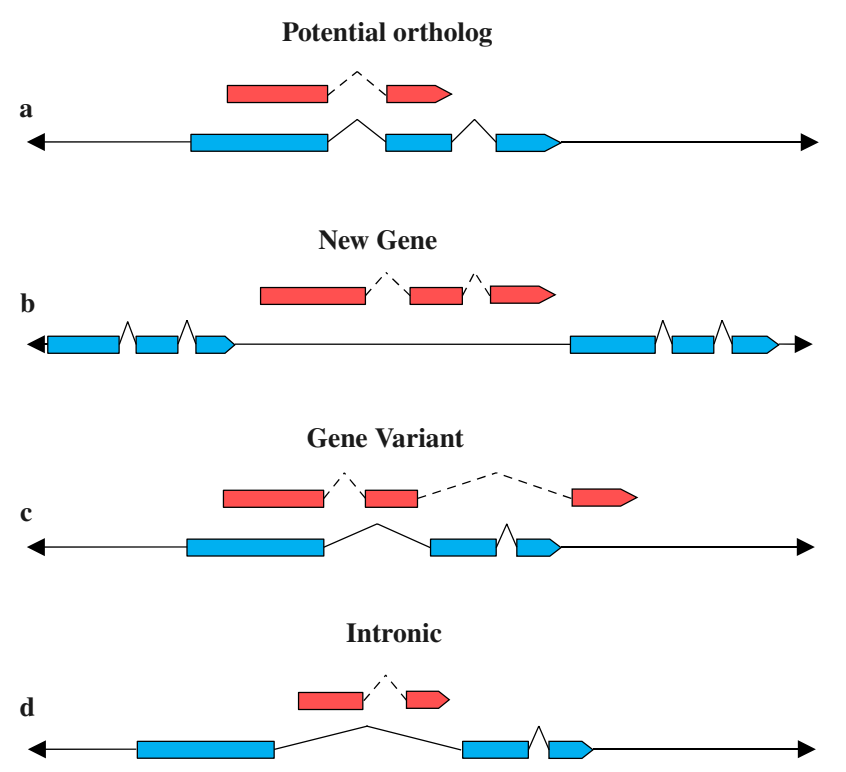

Figure 2

Schematic representation of the four categories for the aligned genes. Red boxes indicate the aligned transcript, blue boxes indicate exons of annotated genes on the genome. a) potential ortholog, b) new gene, c) gene variant, d) intronic.

are classified as novel while from human only $3.5 \%$ of the genes were novel (Table 2).

\section{Manual curation}

The accuracy of the classification generated by the pipeline was manually verified for all the categories, except "contig", for which the annotation information of genomic regions was not available. This process was performed using a web interface tool described in the alignments procedures section of the methods, where the data on the alignments are reported and graphically displayed (Figure 5, [38]). The web interface tool provides a direct external link to the Ensembl website, to allow all the information for a given gene to be easily accessed. Criteria used to evaluate the significance of the alignments, and therefore the correctness of the classification, were: the conservation of gene structure between human and cow, the parameters of alignments and the presence of genscan predictions, ESTs, mRNAs and UniGene features, aligned alongside with the query sequence. Only protein coding genes aligned to the genome with overall identity equal or greater than $75 \%$ were selected for the manual curation, even though the sequences below this threshold had highly significant E-values. The genes in the "not aligned" category, which could not be selected using a threshold, were all examined. The analyses and supporting evidence for each library is presented in the following sections and the results summarised in Table 3.

\section{In silico bovine Libraries \\ Potential Orthologs}

This category had the largest number of genes with at least $75 \%$ of identity, almost $79 \%$ of the total, and contains 883 sequences aligned to exons of known human genes. Those with at least $90 \%$ identity (177) were systematically examined, however, additional random checks were performed on the whole library. In most of the cases $(80 \%)$, bovine sequences were found to be shorter than the corresponding human genes, with bovine transcripts clearly missing one or more exons at the gene boundaries. In addition, for some short cow genes the alignments showed a relationship with nearby genes, suggesting that they might be wrongly annotated as distinct genes, while in fact they represent a single gene. Short genes were also represented by terminal exons of misassembled genes which also showed matches elsewhere in the genome. Many of these short genes have been removed in recent releases of Ensembl (51 and 52 in particular). The comparison between cow and human genomes through these alignments, showed that many cow genes had additional evidence aligned alongside the corresponding gene region(s), such as ESTs, mRNAs and genscans to support the presence of additional exons, which had not been annotated, in the proximity of the bovine genes. In a few cases $(10 \%)$, bovine sequences could be aligned with human pseudogenes or retrotransposed genes. Potentially, real orthologs were identified only for short sequences and other particular situations, such as genes located at the ends of contigs or on unassigned chromosomes that appeared truncated or with wrongly assembled structure. Artefacts (i.e. non informative alignments due to the high percentage of identity but very low coverage of the sequence) and nested genes constituted a minority of this class.

\section{Gene Variants}

The total number of sequences examined in this class was 154 , and for $67 \%$ substantial differences in gene length and structure were observed between cow and human genes. These sequences probably represent species-specific differences in gene architecture. In 13\% of the sequences the presence of additional exons is suggested in the structure of the human gene, which was confirmed by the quality of the alignments and the presence of genscan predictions. The remaining $20 \%$ of the results showed unreliable alignments and are likely to be alignment artefacts resulting from short sequences or short stretched of high homology in otherwise divergent sequence.

\section{New Genes}

The bovine sequences aligned in intergenic regions of the human genome were classified as possible new human genes; of these 112 sequences were examined in detail. Results for $57 \%$ of these annotated genes showed an over- 
Table I: Classification of bovine and human non-orthologous genes in categories according to the alignment results

\begin{tabular}{|c|c|c|c|c|}
\hline & \multicolumn{2}{|c|}{ cow vs. human } & \multicolumn{2}{|c|}{ human vs. cow } \\
\hline & n. of genes & $\%$ of the total & n. of genes & $\%$ of the total \\
\hline aligned genes & 3,390 & 89 & 967 & 96 \\
\hline potential ortholog & 2,533 & 67 & 120 & 12 \\
\hline new gene & 472 & 12 & 500 & 50 \\
\hline gene variant & 289 & 8 & 294 & 29 \\
\hline intronic & 96 & 2 & 43 & 4 \\
\hline contig & - & - & 10 & 1 \\
\hline not aligned & 411 & 11 & 43 & 4 \\
\hline total non-orthologous genes & 3,801 & - & 1,010 & - \\
\hline
\end{tabular}

lap with genscan predictions and EST features that aligned with the same regions of the human genome. Many of these features showed good correspondence with the structure of the bovine gene to which they aligned. The results showed that $41 \%$ of the genes aligned with empty regions of the genome where no other annotated features, such as ESTs or genscans, were present. The remaining 2\% of the sequences were re-classified as potential orthologs as they were annotated as genes in later releases of Ensembl (51 and 52). In particular new human genes were annotated in these later releases at the same position where the pipeline described in this paper had placed the corresponding bovine gene.

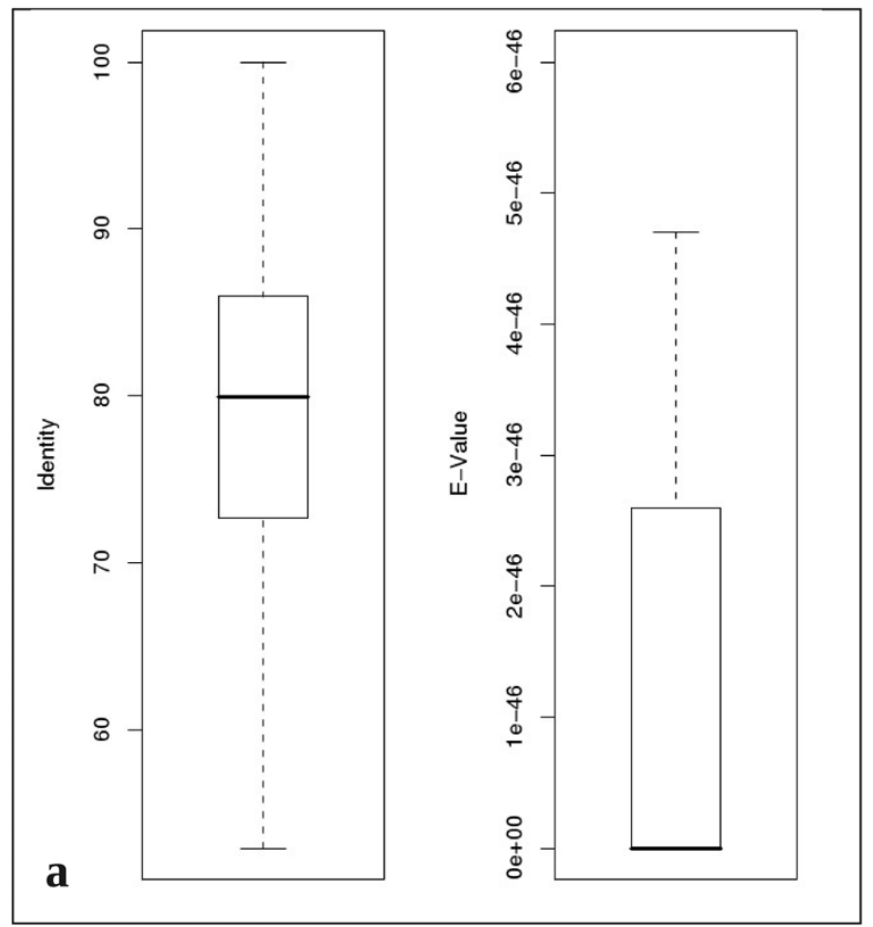

Intronic

Sixteen cow sequences aligned completely within introns of annotated human genes. Of these sequences, $81 \%$ showed an overlap with existing genscan or ESTs features aligned in the same region of the human genome. Overall $19 \%$ of the results showed unreliable alignments.

\section{Not Aligned}

Among the bovine sequences that did not align to the human genome, 273 were protein coding, most (79\%) were annotated as "novel genes" by Ensembl (Table 2). When aligned with BLASTX against the protein nonredundant database at NCBI, $11 \%$ had a hit with human

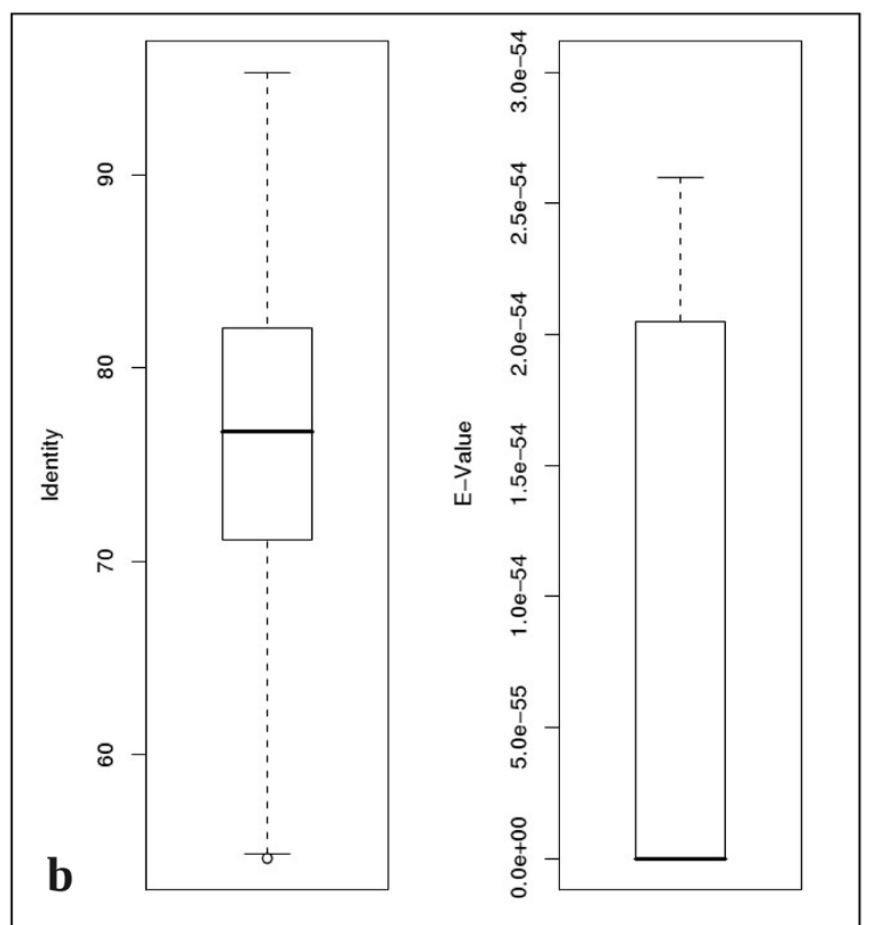

Figure 3

Statistical box plot representing the distribution of identity percentage and E-value of alignments in "cow vs. human" (a) and "human vs. cow" (b) comparisons. 


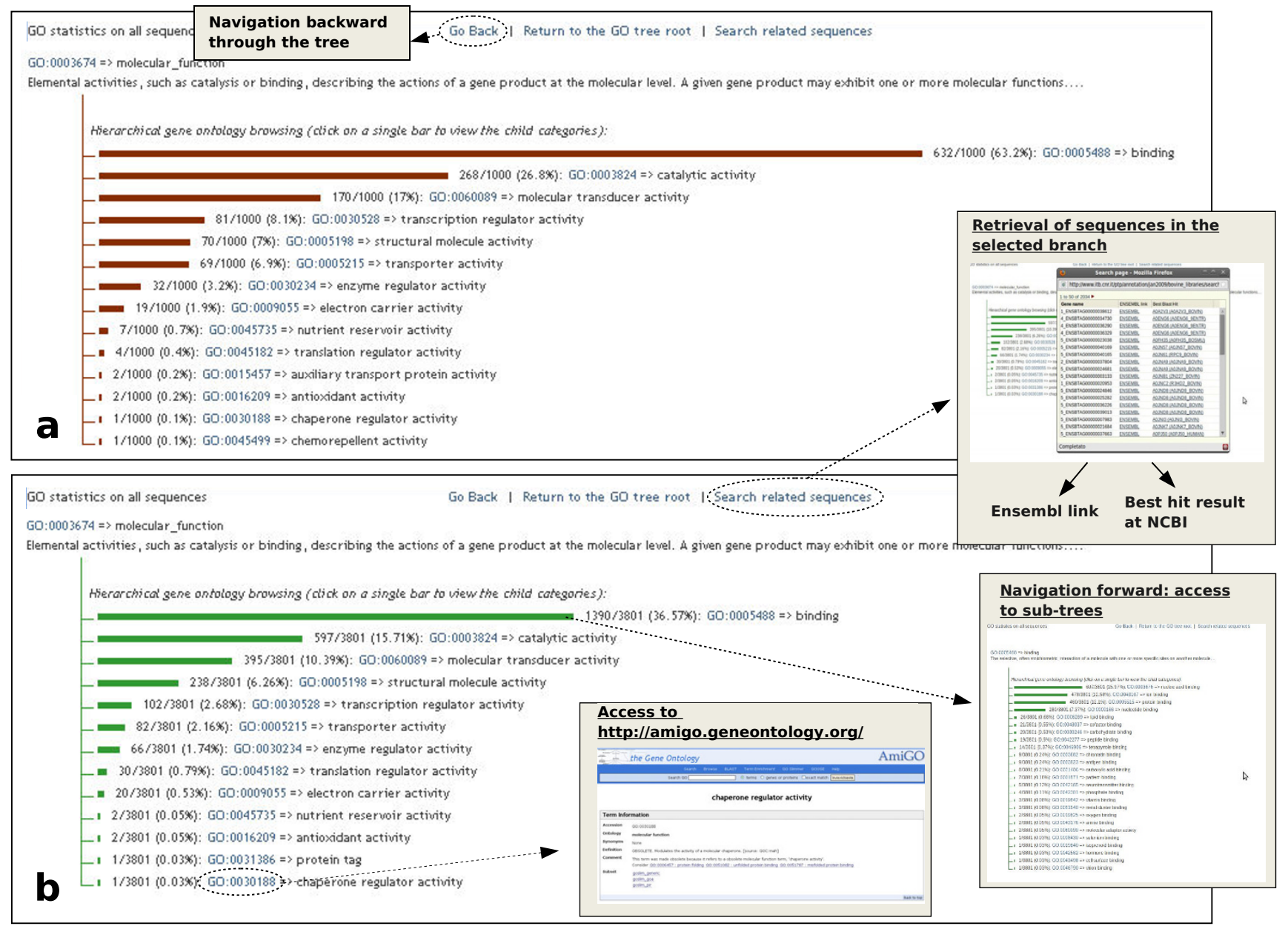

\section{Figure 4}

Tool to display tree-like representation of the GO graphs. Main root for molecular function category is shown for human (a) and bovine (b) libraries. The main navigation features of the web site are indicated by grey boxes.

sequences, BAC clones and synthetic constructs (e.g. plasmids, clones) and $23 \%$ had a match with an E-value lower than 1 e-10 with sequences from other species, such as sheep and goat. In addition, $29 \%$ showed similarity to the nucleotide database, both with human and bovine

Table 2: Status of protein coding genes from the two comparisons

\begin{tabular}{lllll}
\hline & \multicolumn{2}{c}{ cow vs. human } & \multicolumn{2}{c}{ human vs. cow } \\
\cline { 2 - 5 } & known & novel & known & novel \\
\hline potential orthologs & 416 & 705 & 114 & 6 \\
new genes & 70 & 227 & 480 & 20 \\
gene variant & 129 & 131 & 289 & 5 \\
intronic & 8 & 42 & 42 & 1 \\
not aligned & 58 & 215 & 40 & 3 \\
total & 681 & 1,320 & 965 & 35 \\
\end{tabular}

sequences, while the remaining $37 \%$ had no match at all. The problem of finding a significant match within this library is partly due to $25 \%$ of the sequences being less than 100 nucleotides in length, and therefore most are likely to be artefacts, short open reading frames or fragments of sequences, indeed $33 \%$ of them were located on unassigned chromosomes. Ensembl reported no evidence (genscan models, ESTs, UniGene features) for 52\% of these sequences while significant evidence was available only for $12 \%$. The remaining sequences had only partial and unreliable evidence. Some of the sequences showing a good gene structure, but that originally had no match with the protein database, have had protein evidence added in a more recent Ensembl release (version 53). Within this last group only one gene is described as "known", with good supporting evidence and is annotated as "Stella Fragment" (Developmental pluripotencyassociated protein 3, DPPA3; ENSBTAG00000038326). 
Table 3: Results of manual curation.

\begin{tabular}{|c|c|c|c|c|c|c|c|c|}
\hline & \multicolumn{4}{|c|}{ cow vs. human } & \multicolumn{4}{|c|}{ human vs. cow } \\
\hline & $\begin{array}{l}\text { potential } \\
\text { orthologs }\end{array}$ & $\begin{array}{r}\text { gene } \\
\text { variants }\end{array}$ & $\begin{array}{r}\text { new } \\
\text { genes }\end{array}$ & intronic & $\begin{array}{l}\text { potential } \\
\text { orthologs }\end{array}$ & $\begin{array}{r}\text { gene } \\
\text { variants }\end{array}$ & $\begin{array}{r}\text { new } \\
\text { genes }\end{array}$ & intronic \\
\hline pseudogenes and retrotransposed & 17 & & & & & & & \\
\hline potential orthologs & 6 & & 2 & & 13 & & & \\
\hline different length and structure & 142 & 103 & & & 32 & & & \\
\hline additional exons & & 20 & & & & & & \\
\hline unreliable & 12 & 31 & & 3 & 4 & 11 & 105 & 8 \\
\hline overlap Genscan or EST, potential new genes & & & 64 & 13 & & & & \\
\hline no evidence, potential new genes & & & 46 & & & & & \\
\hline overlap different genes & & & & & 16 & & & \\
\hline gene variants & & & & & & 144 & & \\
\hline problems in annotation & & & & & & 25 & 2 & \\
\hline new genes/partial & & & & & & & $125 / 55$ & \\
\hline nested genes & & & & & & & & II \\
\hline examined (>75\% identity) & I77(883) & 154 & 112 & 15 & 65 & 180 & 287 & 19 \\
\hline total & $|12|$ & 260 & 297 & 60 & 120 & 500 & 294 & 43 \\
\hline
\end{tabular}

Number of genes for each category are reported.

The same gene is present in human and mouse but is not classified as orthologous in cow. Even though the annotated bovine gene appears to be a fragment, the sequence diverges significantly from the sequence of the other species.

\section{In silico human libraries}

Potential Orthologs

- Human genes aligned to the bovine genome, and having an overlap only with known exons of annotated genes, were classified as "potential orthologs". In total 65 genes were examined and fell into four different classification groups. Despite the good overlap with annotated bovine exons, $50 \%$ of these sequences were either shorter or longer than the corresponding bovine gene, i.e. had either different number of exons or exons with different length. These genes were most likely not annotated as orthologs because, in the current sequence assemblies, they have species-specific features. In the second case, $24 \%$ of the sequences overlapped two different bovine genes located in the same chromosomal region, or novel bovine genes with a single exon. The presence of bovine genscan predictions and other EST features in the same region suggested annotation or sequence assembly problems with the bovine genome in that particular position. Of the sequences missed by the Ensembl automated annotation pipeline, 20\% could be considered as orthologs as they have a good structure and sequence overlap with bovine genes. The remaining $6 \%$ of the sequences showed a nonreliable alignment (artefacts) or aligned to pseudogenes.

\section{Gene Variants}

Human genes aligned to bovine genome and having an overlap both with exons and introns or upstream/downstream regions of known bovine genes, were classified as "gene variants". Several different examples were identified within the 180 genes examined. The alignment of human gene sequences with the presence of genscan predictions or ESTs features together with the quality of the match, confirmed $80 \%$ of the sequences as potential "gene variants". For $14 \%$ of the sequences, problems with the annotation were observed, e.g. where a human gene matched with 


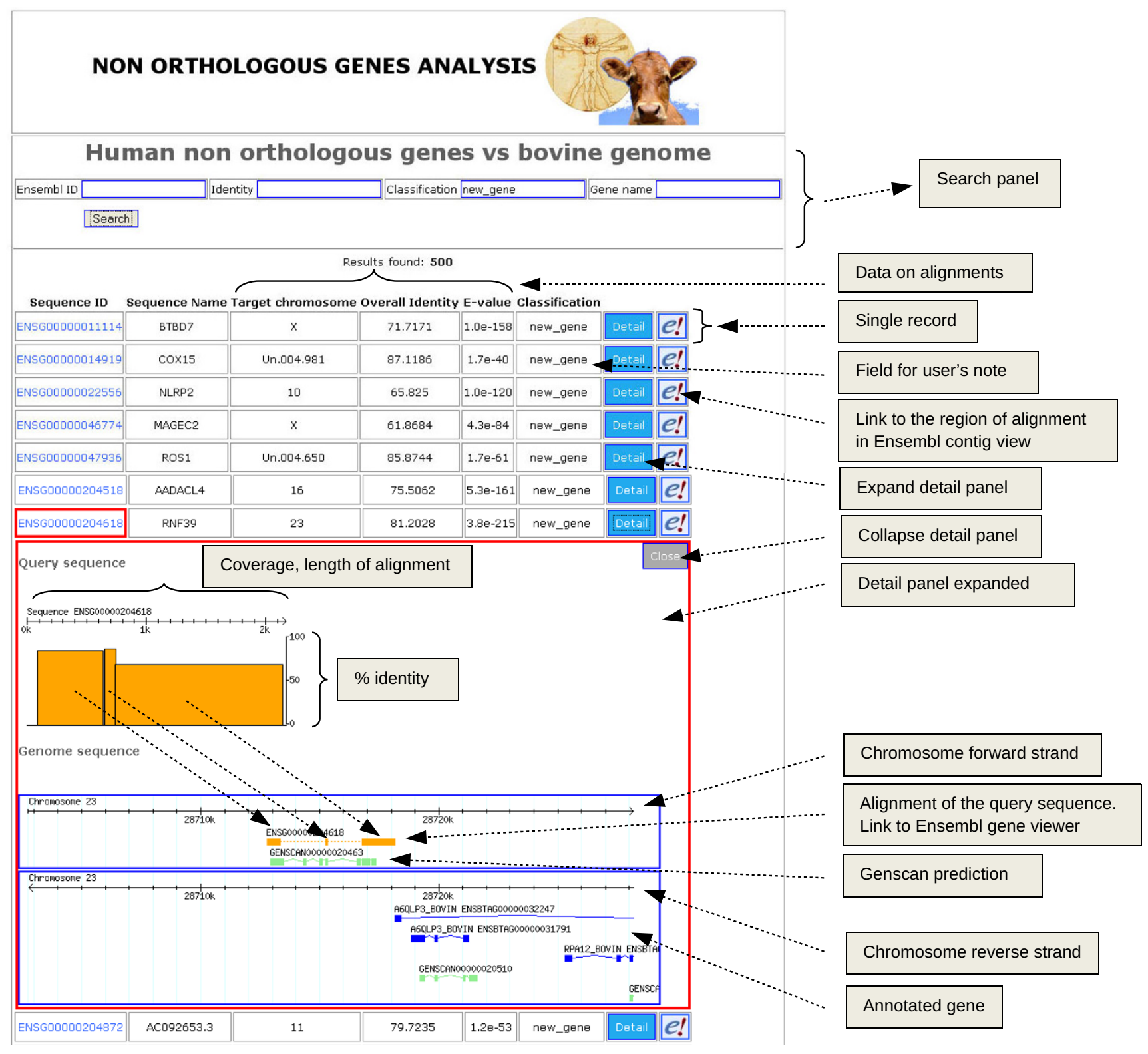

\section{Figure 5}

Web interface to display the results of alignments of the bovine and human libraries. Records can be retrieved by Ensembl ID, percentage of identity of the alignment, classification and gene name, through the search panel. For each record, representing a sequence, the EnsembI ID, the sequence name, the chromosome of the alignment, the percentage of identity, the E-value of the alignment and the classification flag were available. Moreover a link takes the user to the corresponding region of the alignment in the Ensembl contig view and the "detail" button expands a detail panel. In this panel the selected alignment is represented graphically in order to show the coverage and percentage of identity of the sequence but also the position of the alignment to the genome scaffold, alongside with genscan predictions and known gene sequences, retrieved directly from Ensembl database. 
two different bovine genes in the same region; these are probably bovine genes placed on misassembled regions of the genome sequence, or that had been incorrectly annotated as two distinct genes. The remaining $6 \%$ of the results were considered unreliable alignments (i.e. short alignments or sequencing artefacts).

\section{New Genes}

Human sequences aligned to intergenic regions, i.e. where no genes had been identified, were classified as "new genes". This category had 287 protein coding genes of which $44 \%$ could be considered new bovine genes. These findings were also supported by new information introduced in the latest releases of Ensembl (from 51 onward) where, for the $26 \%$ of those sequences verified as potential "new gene" by the manual curation, a new Ensembl feature identified as "EST based gene" was found in the same position corresponding as produced by our pipeline. For $19 \%$ of the "new genes" aligned to genomic regions with supporting evidence such as genscan predictions, ESTs, or mRNAs, the evidence and overlap were incomplete and therefore these sequences were classified as "partial new genes". Similar situations were observed where human genes overlapped two or more genscan predictions, or aligned within very large predicted genes, which suggested problems in the annotation of specific bovine genome regions. The remaining results contained short sequences, fragments and artifacts that produced unreliable alignments. In some cases, human genes aligned with genscan exons that were spaced at large distances on the bovine scaffold, or had specific parts of the gene matching with different regions of the genome. A particular example of this situation was found for olfactory receptors, which are recurrent motifs in the dataset of sequences. These probably represent domains or repeated gene structures showing high similarity with sequences at many different chromosomal regions.

\section{Intronic}

This category includes all the human sequences aligned completely within introns of known bovine genes. A total of 19 sequences were examined and 57\%, were considered possible new nested genes. The remaining $42 \%$ of the results represented artefacts or short aligned sequences (less than 100-150 nucleotides in length).

\section{Not Aligned}

Overall 43 human sequences had no match with the bovine genome. Forty sequences were known genes and three were novel genes (Table 2). These sequences were translated in all six reading frames and BLASTed against the protein non-redundant database: for 35 a match was found with a bovine sequence and in the $29 \%$ of the cases the annotation and description corresponded to that for the human gene.

\section{Discussion}

Over the last decade, many different approaches for identifying gene orthology between species have been proposed in the literature. The process of gene annotation, as well as the discrimination between protein coding and non coding genes [39], will become even more important as the number of available genome sequences increases, in line with the rapid progress of the sequencing technology. Depending on the sensitivity and specificity of methods used to identify orthologous genes, the fraction of genes without orthologs between species is variable, and also depends on the quality of the genome assembly $[40,41]$. Among these genes, there are the so called orphans [32], which have no homologs among the genes of other species. Even though several explanations have been proposed for the absence of homologs, one of the possibilities is that they might represent species specific genes. In the literature, the search for orphans genes has been carried out in different species by comparing gene sets at protein level $[34,42,43]$. In the work presented here we faced the problem of non-orthologous genes between species at nucleotide level. We focused on the bovine genome (version 4.0), whose assembly and annotation is still ongoing. Ensembl orthology predictions from release 50 were used, as these represented the highest quality genome annotations across several mammalian species. Ensembl automatically produces orthology predictions between species and for each release of the database these predictions can be easily queried using the "BioMart" tool. A simple query to obtain the number of non-orthologous genes between the bovine and the human genome returned 5,507 out of 22,836 genes. The reverse query returned 19,811 out of 36,396 human genes with no orthologs in the bovine genome. The differences are dependent on the level and quality of annotation for the two genomes, and on the larger set of annotated human genes. In order to reduce this effect in a simple two-way comparison, the bovine and human datasets were filtered with information coming from other completed genomes, specifically mouse and dog. A total of 3,801 bovine genes had no orthologs with these three species while 1,010 human genes, with orthologs in mouse and dog, had no orthologs in cow (Figure 1). These two groups of genes were considered as the most consistent nonorthologous genes to use in further work. In the previous assembly of the cow genome (Btau 3.1, Ensembl release 49), a similar query gave almost double the number of bovine non-orthologous genes $(6,247)$, while nonorthologous human genes were slightly fewer (865). This reflects major improvements in the bovine genome assembly and annotation between version 3.1 and 4.0, but suggests that there are still problems either with the assembly or the annotation of the bovine sequence.

The two sets of non-orthologous genes (cow vs. human and human $v$ s. cow) were investigated in order to test the 
quality of orthology predictions, to reveal genuine differences between species and most commonly show problems with the genome assemblies. A bioinformatic pipeline and web tool were developed to describe the alignments of each library with the genome of the other species, and the alignments were classified into 5 different categories, according to the annotation associated with the sequence in each genome (Figure 2, Table 1). These classes were established according to the different scenarios that might explain annotation problems, which were: potential orthologs, gene variants, new genes, intronic genes and not-aligned sequences. For this analysis only the protein coding genes were selected, which most likely represent functional genes, while pseudogenes and retrotransposed genes were removed as the non-coding RNAs, which were analysed separately [44].

Although all the aligned sequences showed highly significant E-values, only results with more than $75 \%$ of overall identity were targeted for a detailed manual curation. A web based informatic tool was created and used that provides easy access to the alignments and available annotation for each gene.

Among the genes examined, $90 \%$ of the sequences had a significant match, even though for a small fraction the alignments were not reliable. These included very short sequences and genes which had short alignments or that aligned with two different genes within the same genomic region, and were considered sequence or alignment artefacts. These "problematic" sequences were distributed throughout the genome and did not suggest the presence of localised regions with problems with the genome assembly or annotation (data not shown).

The current level of annotation of the bovine genome is not comparable with that of human, however the alignment of the annotated bovine genes with the human genome produced some interesting results. In some cases there was evidence to suggest new, presently unannotated, features in the human genome, including additional exons, as observed in the "gene variant" class, or potential new human genes, from the "new gene" and "intronic" classes. The latter were supported by the presence of other evidence in the region of the alignments, such as the coincident alignment of EST and genscan predictions. Indeed, some of the features identified appeared in later releases of Ensembl database, where additional human genes have been annotated exactly where the pipeline used here had aligned a bovine gene. This observation supports the value of this type of comparative approach. The "potential ortholog" class helped to identify additional orthology relationships, however, it also identified deficiencies in the genome sequence and errors in the annotation of many bovine genes. Generally, the annotation suggested that cow genes were shorter than the human orthologous genes, which in many cases was because exons had been missed at gene boundaries. Alignment of EST and genscan predictions, in the corresponding positions of the bovine genome, suggested the presence of new bovine exons. In addition many genes were identified in the bovine genome that had not been annotated.

It would be expected that genes with orthologs in human, mouse and dog should have homology relationships in cow, even though they had not been identified by the automated orthology prediction. Thus, the alignment of the human genes to the bovine genome should find new features to improve genome annotation in cow. From the results in the "new gene" class, $46 \%$ could be considered as new bovine genes, indeed in latest Ensembl releases half of those identified using the approach described here were added in a new Ensembl feature called "EST based genes", which were in agreement with our alignments. The interpretation of the results for genes in the "potential ortholog", "gene variant" and "intronic" classes, becomes more complex as it is not completely clear if the observed alignments and differences are due to species-specific features, or problems with the bovine annotation or the genome assembly. From the genes belonging to "potential orthologs", 20\% may be considered as true orthologs which were missed by the Ensembl prediction pipeline, for the most part due to minor differences between the sequences. Accepting the current annotation of the bovine genome, $80 \%$ of the results in the "gene variant" class were highlighted the presence of new exons for genes currently annotated in cow.

The "not aligned" class may contain real non-orthologs between the four species but also orphan genes with no match with other species. This class was analysed for both cow and human genomes, by searching similarities with the complete non-redundant protein database from NCBI. For most of the human sequences, a match was identified with bovine proteins whose annotation and description is exactly the same as in human. These results most probably represent gene sequences that are still not annotated or assembled into the bovine genome, and hence were completely missed by the Ensembl orthology prediction. Some of the cow genes for which there is no match with the human genome may be indeed novel, bovine orphan genes, as only $11 \%$ in this class had a significant match with a human sequence and $37 \%$ had no match at all in the NCBI database. Among these genes there are novel sequences which also have supporting protein evidence; these are interesting candidates among which to look for cow specific coding regions. The functions of orphan genes are generally poorly characterized [43], they show distinctive features such as high tissue specificity, rapid evolution and short peptide size [34]. 
Recent works have demonstrated that they evolve three to four times faster than the average genes in Drosophila [43] and in primates [34]. In some cases the sequence divergence between species may be so great that the orthology between the genes is not obvious. This situation is represented by the "Stella fragment" related gene (DPPE3), which is annotated and has good supporting evidence. Indeed this gene has human and mouse counterparts but with the sequences highly divergent between the species.

The discrimination between orthologs and paralogs still remains difficult, especially when comparing incomplete and large genomes, as addressed by Fulton et al. [45]. Genes predicted as paralogs by Ensembl are $49 \%$ and 60\% of the bovine and human libraries, respectively. Paralogs, which mainly arise from a duplication event and may undergo structural rearrangements during evolution [1], are found in the non-orthologous sets described herein. Their sequence divergence might explain why they were missed as orthologs between species and in some cases can be traced back with the similarity approach used in this work.

Ontology descriptions, even if not complete for the bovine gene set, due to the lower level of annotation, were interesting in describing the groups of genes created in this work. Many of the genes with no apparent orthologs were clustered as proteins with binding properties. The typical modular composition of such proteins and their specificity for different ligands could explain structural differences which might have an effect on the orthology prediction. Despite the annotation and similarity search performed to retrieve GO terms for the bovine non orthologous genes, no valid annotation was found for the 75\% of the cow genes in the "not aligned" group. This highlights the need to focus on this particular group of genes that might reveal orphan as well as species specific coding sequences.

\section{Conclusions}

This study was focused on a particular class of genes predicted to be non-orthologous between cow and human genomes. These genes are normally considered the result of divergent evolution and are reported by the automated pipelines and following the manual annotations of the bovine and dog genome in the international sequencing projects $[31,36]$. Evidence found within this work suggest that a high number of non-orthologous genes between cow and human could be considered a side effect of an incomplete genome assembly and annotation process. The approach described here allowed the differences and similarities for this class of genes to be highlighted and also possible new features for the human genome to be identified. The comparison between species, using simi- larity and classification methods, is crucial for the analysis of genome sequences and gene sets, especially when the annotation process relies on the quantity and quality of available data on transcript sequences for a particular species. In this context, this kind of comparative approach could be used to extend the current genome annotation protocols. The presence of non-orthologous genes in other species should be considered as a central resource to derive important information for the definition of gene models and structures, in particular for the newly assembled genomes, where the lack of a complete set of genes and poor transcript sequences information may restrict the commonly applied annotation procedures.

\section{Methods \\ Datasets}

The cow genome assembly version 4.0 and the human genome assembly version 36 were downloaded from Ensembl release 50 [46]. Gene sets were retrieved directly from Ensembl database using the multi-species comparison options in the BioMart web interface [47]. In detail, the bovine library of non-orthologous genes was obtained by retrieving three sets of Gene IDs obtained after applying a filter to exclude the orthologous genes of human, mouse and dog, respectively 5,507, 5,273 and 5,842 genes. Only the genes in common among these three sets $(3,801)$ were retained (Figure 1a). The second library is referred as "human"; this refers to the human subset of genes that are found in common between human, mouse and dog genomes and represent core mammalian orthologs. Two lists of human genes were built; the first contained orthologs between human and mouse datasets filtered to exclude the orthologous genes of cow and the second by the orthologs between human and dog datasets, filtered to exclude the orthologous genes of cow. Only human Gene IDs in common between these two lists (respectively 2,104 and 1,437 genes) were retained for a total of 1,010 genes (Figure 1b). All the possible orthology predictions (1:1, many:1, 1:many, many:many) as provided by Ensembl were used.

The protein and nucleotide non-redundant databases were downloaded from NCBI on January 2009 [48].

\section{Statistical plots}

Statistical box plots and distributions were drawn using $\mathrm{R}$ v2.7.0 [49]. In this analysis, the best hit with the lowest Evalue for each sequence was considered.

\section{Alignment procedure}

All the alignments were done using WU-BLAST v2.0 [50], with identity matrix for nucleotide alignments and BLOSUM62 identity matrix for protein alignments. The longest transcript (canonical transcript) for each gene was aligned to the whole genome sequence using the parame- 
ters $\mathrm{W}=10, \mathrm{E}=1 \mathrm{e}-5$, links, topcomboN $=1$ and hspsepS$\max =1,000,000$. The best hit with the lowest E-value was considered as the result for each alignment. Custom Perl scripts were written to run the alignments, parse the results using BioPerl v1.5.2 libraries [51] and store the information into a relational MySQL v5.0 database [52]. The results were classified according to the genomic position and to the current annotation available for each species, using the Ensembl Perl API to query the Ensembl database and retrieve all the genomic information $[27,53]$. A web interface was created to query the database of results and display the aligned sequences and the corresponding genomic regions, with other annotated genes and the genscan [54] predictions available. This interface was written using the Ruby language and was based on the Ruby on Rails framework [55]. The interface was used to perform the manual annotation and visual inspection of the results, providing the possibility to query the database of results directly using different parameters, such as alignment identities, gene IDs and classification labels. Direct access to the Ensembl information for each gene was added to help the retrieval of all the necessary information during the manual annotation phase. The interface is available at http://www.itb.cnr.it/idralab/ non orthologs.

\section{Gene Ontology (GO) Analysis}

We obtained Human GO annotations via the Biomart searching tool from Ensembl release 52 [46,47]. The Gene Ontology Database (January, 2009)[56,57] was used to extend this preliminary GO data set with all the "is-a" related GO terms (e.g. GO:0004601 - peroxidase activity "is a" GO:00016209 - antioxidant activity), up to the three main roots (molecular function, cellular component and biological process) in the Gene Ontology hierarchy.

Bovine GO annotations derive from the EBI Gene Ontology Annotation (GOA) database (January, 2009) [58,59], without distinguishing between the available evidence codes. In details, sequences were blasted (BLASTX, e-value threshold of 1e-10) against a modified version of the UniprotKB database (January, 2009)[60,61] containing only GOA annotated proteins, and were linked to GO annotations in accordance with the GOA database. As in the case of human sequences, "is-a" related GO terms were added, according to the GO DAG available data.

Perl scripts were designed to produce library-specific statistics on the sequence distribution among the GO terms, and to save the data into a relational MySQL v 5.0 database [52].

A software tool written in PHP language was developed in order to display the sequence-GO distribution. This visualization tool dynamically creates a navigable, tree-like representation of the GO graph, showing each GO term as a bar proportionally long as the number of the related sequences (Figure 4). The visualisation tool is available at http://www.itb.cnr.it/ptp/annotation.

\section{Authors' contributions}

RM and FS carried out the bioinformatic analyses and wrote the paper; AC performed the GO analysis; PAM and JLW critically reviewed the paper. All the authors read and approved the final manuscript.

\section{Acknowledgements}

We acknowledge the Laboratory of Interdisciplinary Technologies in Bioinformatics (L.I.T.B.I.O.) project for the computational infrastructure http:// www.litbio.eul. FS and JLW acknowledge the support of the Cariplo Foundation.

\section{References}

I. Koonin EV: Orthologs, paralogs, and evolutionary genomics. Annu Rev Genet 2005, 39:309-338.

2. Studer RA, Robinson-Rechavi M: How confident can we be that orthologs are similar, but paralogs differ? Trends Genet 2009, 25:210-216.

3. Zmasek CM, Eddy SR: RIO: analyzing proteomes by automated phylogenomics using resampled inference of orthologs. $B M C$ Bioinformatics 2002, 3: 14.

4. Storm CEV, Sonnhammer ELL: Comprehensive analysis of orthologous protein domains using the HOPS database. Genome Res 2003, 13:2353-2362.

5. Storm CEV, Sonnhammer ELL: Automated ortholog inference from phylogenetic trees and calculation of orthology reliability. Bioinformatics 2002, 18:92-99.

6. Dehal PS, Boore JL: A phylogenomic gene cluster resource: the Phylogenetically Inferred Groups (PhIGs) database. BMC Bioinformatics 2006, 7:201.

7. Goodstadt L, Ponting CP: Phylogenetic reconstruction of orthology, paralogy, and conserved synteny for dog and human. PLoS Comput Biol 2006, 2: el 33.

8. Li H, Coghlan A, Ruan J, Coin LJ, Hériché J, Osmotherly L, Li R, Liu T, Zhang Z, Bolund L, Wong GK, Zheng W, Dehal P, Wang J, Durbin R: TreeFam: a curated database of phylogenetic trees of animal gene families. Nucleic Acids Res 2006, 34:D572-D580.

9. Wall DP, Fraser HB, Hirsh AE: Detecting putative orthologs. Bioinformatics 2003, 19:1710-1711.

10. Deluca TF, Wu I, Pu J, Monaghan T, Peshkin L, Singh S, Wall DP: Roundup: a multi-genome repository of orthologs and evolutionary distances. Bioinformatics 2006, 22:2044-2046.

I I. Altschul SF, Madden TL, Schäffer AA, Zhang J, Zhang Z, Miller W, Lipman DJ: Gapped BLAST and PSI-BLAST: a new generation of protein database search programs. Nucleic Acids Res 1997, 25:3389-3402.

12. Tatusov RL, Fedorova ND, Jackson JD, Jacobs AR, Kiryutin B, Koonin EV, Krylov DM, Mazumder R, Mekhedov SL, Nikolskaya AN, Rao BS, Smirnov S, Sverdlov AV, Vasudevan S, Wolf YI, Yin JJ, Natale DA: The COG database: an updated version includes eukaryotes. BMC Bioinformatics 2003, 4:4I.

13. Tatusov RL, Natale DA, Garkavtsev IV, Tatusova TA, Shankavaram UT, Rao BS, Kiryutin B, Galperin MY, Fedorova ND, Koonin EV: The COG database: new developments in phylogenetic classification of proteins from complete genomes. Nucleic Acids Res 200I, 29:22-28

14. Lee Y, Sultana R, Pertea G, Cho J, Karamycheva S, Tsai J, Parvizi B, Cheung F, Antonescu V, White J, Holt I, Liang F, Quackenbush J: Cross-referencing eukaryotic genomes: TIGR Orthologous Gene Alignments (TOGA). Genome Res 2002, I 2:493-502.

15. Remm M, Storm CE, Sonnhammer EL: Automatic clustering of orthologs and in-paralogs from pairwise species comparisons. J Mol Biol 200 I, 3 I 4: I04 I-I052. 
16. O'Brien KP, Remm M, Sonnhammer ELL: Inparanoid: a comprehensive database of eukaryotic orthologs. Nucleic Acids Res 2005, 33:D476-D480.

17. Alexeyenko A, Tamas I, Liu G, Sonnhammer ELL: Automatic clustering of orthologs and inparalogs shared by multiple proteomes. Bioinformatics 2006, 22:e9-I5.

18. Enright AJ, Dongen SV, Ouzounis CA: An efficient algorithm for large-scale detection of protein families. Nucleic Acids Res 2002 , 30: $1575-1584$

19. Li L, Stoeckert CJ, Roos DS: OrthoMCL: identification of ortholog groups for eukaryotic genomes. Genome Res 2003, 13:2178-2189.

20. Chen F, Mackey AJ, Stoeckert CJ, Roos DS: OrthoMCL-DB: querying a comprehensive multi-species collection of ortholog groups. Nucleic Acids Res 2006, 34:D363-D368.

21. Kanehisa M, Goto S, Kawashima S, Okuno Y, Hattori M: The KEGG resource for deciphering the genome. Nucleic Acids Res 2004 32:D277-D280.

22. Fu Z, Chen X, Vacic V, Nan P, Zhong Y, jiang T: MSOAR: a highthroughput ortholog assignment system based on genome rearrangement. J Comput Biol 2007, I 4: I I60-I I75.

23. Schneider A, Dessimoz C, Gonnet GH: OMA Browser--exploring orthologous relations across 352 complete genomes. Bioinformatics 2007, 23:2180-2182.

24. Wheeler DL, Barrett T, Benson DA, Bryant SH, Canese K, Chetvernin V, Church DM, Dicuccio M, Edgar R, Federhen S, Feolo M, Geer LY, Helmberg W, Kapustin Y, Khovayko O, Landsman D, Lipman DJ, Madden TL, Maglott DR, Miller V, Ostell J, Pruitt KD, Schuler GD, Shumway M, Sequeira E, Sherry ST, Sirotkin K, Souvorov A, Starchenko G, Tatusov RL, Tatusova TA, Wagner L, Yaschenko E: Database resources of the National Center for Biotechnology Information. Nucleic Acids Res 2008, 36:DI3-D2 I.

25. Chen F, Mackey AJ, Vermunt JK, Roos DS: Assessing performance of orthology detection strategies applied to eukaryotic genomes. PLOS ONE 2007, 2:e383.

26. Koski LB, Golding GB: The closest BLAST hit is often not the nearest neighbor. I Mol Evol 200I, 52:540-542.

27. Curwen V, Eyras E, Andrews TD, Clarke L, Mongin E, Searle SMJ, Clamp M: The Ensembl automatic gene annotation system. Genome Res 2004, I4:942-950.

28. Vilella AJ, Severin J, Ureta-Vidal A, Heng L, Durbin R, Birney E: EnsemblCompara GeneTrees: Complete, duplication-aware phylogenetic trees in vertebrates. Genome Res 2009 , I 9:327-335.

29. Eyras E, Reymond A, Castelo R, Bye JM, Camara F, Flicek P, Huckle EJ, Parra G, Shteynberg DD, Wyss C, Rogers J, Antonarakis SE, Birney $E$, Guigo R, Brent MR: Gene finding in the chicken genome. $B M C$ Bioinformatics 2005, 6:131.

30. Guigo R, Dermitzakis ET, Agarwal P, Ponting CP, Parra G, Reymond A, Abril JF, Keibler E, Lyle R, Ucla C, Antonarakis SE, Brent MR: Comparison of mouse and human genomes followed by experimental verification yields an estimated 1,019 additional genes. Proc Natl Acad Sci USA 2003, I 00: | | 40- I | 45.

31. The Bovine Genome Sequencing and Analysis Consortium, Elsik CG Tellam RL, Worley KC, Gibbs RA, Muzny DM, Weinstock GM, Adelson DL, Eichler EE, Elnitski L, Guigó R, Hamernik DL, Kappes SM, Lewin HA, Lynn DJ, Nicholas FW, Reymond A, Rijnkels M, Skow LC, Zdobnov EM, Schook L, Womack J, Alioto T, Antonarakis SE, Astashyn A, Chapple CE, Chen H, Chrast J, Câmara F, Ermolaeva O, Henrichsen $C N$, et al:: The genome sequence of taurine cattle: a window to ruminant biology and evolution. Science 2009, 324:522-528.

32. Fisher D, Eisenberg D: Finding families for genomic ORFans. Bioinformatics 1999, I 5(9):759-762.

33. Domazet-Loso T, Tautz D: An Evolutionary Analysis of Orphan Genes in. Drosophila Genome Res 2009, I3:2213-2219.

34. Toll-Riera M, Bosch N, Bellora N, Castelo R, Armengol L, Estivill X Albà MM: Origin of primate orphan genes: a comparative genomics approach. Mol Biol Evol 2009, 26:603-6I2.

35. Mouse Genome Sequencing Consortium, Waterston RH, Lindblad Toh K, Birney E, Rogers J, Abril JF, Agarwal P, Agarwala R, Ainscough $\mathrm{R}$, Alexandersson M, An P, Antonarakis SE, Attwood J, Baertsch R, Bailey J, Barlow K, Beck S, Berry E, Birren B, Bloom T, Bork P, Botcherby M, Bray N, Brent MR, Brown DG, Brown SD, Bult C, Burton J, Butler J, Campbell RD, et al.: Initial sequencing and comparative analysis of the mouse genome. Nature 2002, 420:520-562.
36. Lindblad-Toh K, Wade CM, Mikkelsen TS, Karlsson EK, Jaffe DB, Kamal M, Clamp M, Chang JL, Kulbokas EJ, Zody MC, Mauceli E, Xie $X$, Breen M, Wayne RK, Ostrander EA, Ponting CP, Galibert F, Smith DR, Dejong PJ, Kirkness E, Alvarez P, Biagi T, Brockman W, Butler J, Chin C, Cook A, Cuff J, Daly MJ, DeCaprio D, Gnerre S, et al: Genome sequence, comparative analysis and haplotype structure of the domestic dog. Nature 2005, 438:803-8I9.

37. Gene Ontology website for the present paper [http:// www.itb.cnr.it/ptp/annotation]

38. Non orthologous gene analysis website for the current paper [http://www.itb.cnr.it/idralab/non orthologs]

39. Clamp M, Fry B, Kamal M, Xie X, Cuff J, Lin MF, Kellis M, LindbladToh K, Lander ES: Distinguishing protein-coding and noncoding genes in the human genome. Proc Natl Acad Sci USA 2007, 104:19428-19433.

40. Siew N, Fischer D: Analysis of singleton ORFans in fully sequenced microbial genomes. Proteins 2003, 53:24I-25I.

4l. Zhang G, Wang H, Shi J, Wang X, Zheng H, Wong GK, Clark T, Wang $W$, Wang J, Kang L: Identification and characterization of insect-specific proteins by genome data analysis. BMC Genomics 2007, 8:93.

42. Rubin GM, Yandell MD, Wortman JR, Miklos GLG, Nelson CR, Hariharan IK, Fortini ME, Li PW, Apweiler R, Fleischmann W, Cherry JM, Henikoff S, Skupski MP, Misra S, Ashburner M, Birney E, Boguski MS, Brody T, Brokstein P, Celniker SE, Chervitz SA, Coates D, Cravchik A, Gabrielian A, Galle RF, Gelbart WM, George RA, Goldstein LS Gong F, Guan P, Harris NL, Hay BA, Hoskins RA, Li J, Li Z, Hynes RO, Jones S], Kuehl PM, Lemaitre B, Littleton JT, Morrison DK, Mungall C O'Farrell PH, Pickeral OK, Shue C, Vosshall LB, Zhang J, Zhao O Zheng $\mathrm{XH}$, Lewis S: Comparative genomics of the eukaryotes. Science 2000, 287:2204-22I5.

43. Tay S, Blythe J, Lipovich L: Global discovery of primate-specific genes in the human genome. Proc Natl Acad Sci USA 2009 106: I2019-12024.

44. Strozzi F, Mazza R, Malinverni R, Williams JL: Annotation of $\mathbf{3 9 0}$ bovine miRNA genes by sequence similarity with other species. Anim Genet 2009, 40:125.

45. Fulton DL, Li YY, Laird MR, Horsman BGS, Roche FM, Brinkman FSL: Improving the specificity of high-throughput ortholog prediction. BMC Bioinformatics 2006, 7:270.

46. Hubbard TJP, Aken BL, Ayling S, Ballester B, Beal K, Bragin E, Brent S, Chen Y, Clapham P, Clarke L, Coates G, Fairley S, Fitzgerald S, Fernandez-Banet J, Gordon L, Graf S, Haider S, Hammond M, Holland R, Howe K, Jenkinson A, Johnson N, Kahari A, Keefe D, Keenan S, Kinsella R, Kokocinski F, Kulesha E, Lawson D, Longden I, Megy K, Meid P, Overduin B, Parker A, Pritchard B, Rios D, Schuster M, Slater G, Smedley D, Spooner W, Spudich G, Trevanion S, Vilella A, Vogel J, White S, Wilder S, Zadissa A, Birney E, Cunningham F, Curwen V, Durbin R, Fernandez-Suarez XM, Herrero J, Kasprzyk A, Proctor G, Smith J, Searle S, Flicek P: Ensembl 2009. Nucleic Acids Res 2009, 37:D690-D697.

47. Kasprzyk A, Keefe D, Smedley D, London D, Spooner W, Melsopp C, Hammond M, Rocca-Serra P, Cox T, Birney E: EnsMart: a generic system for fast and flexible access to biological data. Genome Res 2004, I 4:160-169.

48. NCBI data repository for BLAST formatted database [ftp:// ftp.ncbi.nih.gov/blast/]

49. R Development Core Team: R: A language and environment for statistical computing. [http://www.R-project.org]. R Foundation for Statistical Computing. Vienna, Austria ISBN 3-90005I-07-0, URL

50. Lopez R, Silventoinen V, Robinson S, Kibria A, Gish W: WU-Blast2 server at the European Bioinformatics Institute. Nucleic Acids Res 2003, 3 I:3795-3798.

5I. Stajich JE, Block D, Boulez K, Brenner SE, Chervitz SA, Dagdigian C Fuellen G, Gilbert JGR, Korf I, Lapp H, Lehväslaiho H, Matsalla C, Mungall CJ, Osborne BI, Pocock MR, Schattner P, Senger M, Stein LD, Stupka E, Wilkinson MD, Birney E: The Bioperl toolkit: Perl modules for the life sciences. Genome Res 2002, I 2:1611-1618.

52. MySQL website [http://dev.mysql.com/]

53. Stabenau A, McVicker G, Melsopp C, Proctor G, Clamp M, Birney E. The Ensembl core software libraries. Genome Res 2004, 14:929-933.

54. Burge C, Karlin S: Prediction of complete gene structures in human genomic DNA. J Mol Biol 1997, 268:78-94

55. Ruby on Rails website [http://rubyonrails.org/] 
56. Ashburner M, Ball CA, Blake JA, Botstein D, Butler H, Cherry JM, Davis AP, Dolinski K, Dwight SS, Eppig JT, Harris MA, Hill DP, IsselTarver L, Kasarskis A, Lewis S, Matese JC, Richardson JE, Ringwald M, Rubin GM, Sherlock G: Gene ontology: tool for the unification of biology. The Gene Ontology Consortium. Nat Genet 2000, 25:25-29.

57. The Gene Ontology Database [http://archive.geneontology.org/ full/2009-0I-0I/go 20090I-termdb.rdf-xml.gz]

58. Barrell D, Dimmer E, Huntley RP, Binns D, O'Donovan C, Apweiler R: The GOA database in 2009--an integrated Gene Ontology Annotation resource. Nucleic Acids Res 2009, 37:D396-D403.

59. The EBI Gene Ontology Annotation (GOA) database [ftp:// ftp.ebi.ac.uk/pub/databases/GO/goa/UNIPROT/ gene association.goa uniprot.gz]

60. Consortium U: The Universal Protein Resource (UniProt) 2009. Nucleic Acids Res 2009, 37:DI69-DI74.

61. The UniprotKB database [ftp://ftp.uniprot.org/pub/databases/ uniprot/current release/knowledgebase/complete/ uniprot trembl.fasta.gz]

Publish with Bio Med Central and every scientist can read your work free of charge

"BioMed Central will be the most significant development for disseminating the results of biomedical research in our lifetime. "

Sir Paul Nurse, Cancer Research UK

Your research papers will be:

- available free of charge to the entire biomedical community

- peer reviewed and published immediately upon acceptance

- cited in PubMed and archived on PubMed Central

- yours - you keep the copyright

Submit your manuscript here:

http://www.biomedcentral.com/info/publishing_adv.asp
BiolMedcentral 